\title{
Impaired Neurocognitive Functions Affect Social Learning Processes in Oppositional Defiant Disorder and Conduct Disorder: Implications for Interventions
}

\author{
Walter Matthys • Louk J. M. J. Vanderschuren • \\ Dennis J. L. G. Schutter · John E. Lochman
}

Published online: 12 July 2012

(C) Springer Science+Business Media, LLC 2012

\begin{abstract}
In this review, a conceptualization of oppositional defiant (ODD) and conduct disorder (CD) is presented according to which social learning processes in these disorders are affected by neurocognitive dysfunctions. Neurobiological studies in ODD and CD suggest that the ability to make associations between behaviors and negative and positive consequences is compromised in children and adolescents with these disorders due to reduced sensitivity to punishment and to reward. As a result, both learning of appropriate behavior and learning to refrain from inappropriate behavior may be affected.
\end{abstract}

\section{W. Matthys}

Department of Child and Adolescent Psychiatry, Rudolf Magnus Institute of Neuroscience, University Medical Centre Utrecht, Heidelberglaan 100, 3584 CX Utrecht, The Netherlands

e-mail: w.matthys@uu.nl

W. Matthys

Department of Child and Adolescent Studies, Utrecht University, Utrecht, The Netherlands

\section{J. M. J. Vanderschuren}

Department of Neuroscience and Pharmacology, Rudolf Magnus Institute of Neuroscience, University Medical Centre Utrecht, Utrecht, The Netherlands

\section{J. M. J. Vanderschuren}

Division of Behavioural Neuroscience, Department of Animals in Science and Society, Faculty of Veterinary Medicine, Utrecht University, Utrecht, The Netherlands

\section{J. L. G. Schutter}

Department of Experimental Psychology, Utrecht University, Utrecht, The Netherlands

\section{J. E. Lochman ( $\square)$}

Department of Psychology, University of Alabama, Tuscaloosa, AL, USA

e-mail: jlochman@as.ua.edu
Likewise, problem solving is impaired due to deficiencies in inhibition, attention, cognitive flexibility, and decision making. Consequently, children and adolescents with ODD and $\mathrm{CD}$ may have difficulty learning to optimize their behavior in changeable environments. This conceptualization of ODD and CD is relevant for the improvement of the effect of psychological treatments. Behavioral and cognitive-behavioral interventions that have been shown to be modestly effective in ODD and CD are based on social learning. Limited effectiveness of these interventions may be caused by difficulties in social learning in children and adolescents with ODD and CD. However, although these impairments have been observed at a group level, the deficits in reward processing, punishment processing, and cognitive control mentioned above may not be present to the same extent in each individual with ODD and CD. Therefore, the neurocognitive characteristics in children and adolescents with ODD and CD should be assessed individually. Thus, instead of delivering interventions in a standardized way, these programs may benefit from an individualized approach that depends on the weaknesses and strengths of the neurocognitive characteristics of the child and the adolescent.

Keywords Oppositional defiant disorder . Conduct disorder - Neurocognitive dysfunctions . Social learning processes

\section{Introduction}

The characteristic features of oppositional defiant disorder (ODD) and conduct disorder (CD) (American Psychiatric Association 2000) are oppositional, aggressive, and antisocial behaviors. ODD and $\mathrm{CD}$ are among the most 
prevalent psychiatric disorders in children and adolescents, with percentages of 3.2 for ODD and 2.0 for CD (Lahey et al. 1999). In addition to the negative developmental consequences of ODD and CD for the individual, such as poor interpersonal and school adjustment, delinquency, substance use disorders, and other psychiatric disorders (Kim-Cohen et al. 2003; Maughan and Rutter 2001), these disorders also incur high costs to society (Raaijmakers et al. 2011; Scott et al. 2001). Conceptual developmental models are needed as they can identify active mechanisms, which can be targets of preventive and treatment interventions for ODD and CD youth (Matthys and Lochman 2010).

While some models focus on environmental factors such as coercive parent-child interactions (Reid et al. 2002), others also target individual psychological factors such as in the contextual social cognitive model (Lochman and Wells 2002). Such models describe the development of oppositional, aggressive, and antisocial behaviors from the perspective of deviances in social learning based on operant conditioning, that is, the contingencies of reinforcement (Kazdin 2005), and of observational learning, that is, observing another individual engage in behavior without performing the behavior oneself (Bandura 1973). Likewise, these models serve as a foundation for learning-based interventions, that is, behavioral parent training and cognitive behavior therapy, with small-to-medium effect sizes (McCart et al. 2006). Although individual biologically based factors such as temperamental characteristics are acknowledged in these social learning models (e.g., Patterson 2002), in our view, the specific role of neurobiological factors involved in the learning processes resulting in ODD and CD symptoms has been neglected. Here, we present a conceptualization of ODD and CD according to which social learning processes in ODD and $\mathrm{CD}$ are affected by neurobiological factors.

Our conceptual framework consists of three interrelated mental domains: punishment processing, reward processing, and cognitive control (for similar models, see Ernst and Fudge 2009; van Honk et al. 2010). The mental domains in this framework are defined in terms of their functions (e.g., the processing of punishment cues) that are physically realized by the various neurobiological systems (e.g., the amygdala, the autonomic nervous system, and the hypothalamus-pituitary-adrenal axis). According to this conceptual framework presented here, adequate functioning of the three mental domains is necessary for the occurrence of appropriate social learning processes. Indeed, children need to be sensitive to punishment cues in order to learn refraining from inappropriate behaviors. Likewise, normative sensitivity to reward cues is a prerequisite condition for learning appropriate behaviors. Finally, in order to adapt behavior in changeable environments, adequate cognitive control of emotions, thought, and behavior is necessary.

The present conceptualization is based on our recent review in which we provide a coherent picture of the neurobiology of ODD and CD within the framework of these three interrelated mental domains (Matthys et al. 2012). In extension of this review, here we discuss the main findings of this review from the perspective of social learning processes that are impeded in ODD and CD by impairments in the three mental domains. Thus, in the present paper, we first give a concise overview of the neurobiology of ODD and CD from the perspective of the three mental domains. We then conceptualize ODD and $\mathrm{CD}$ as disorders in which social learning processes are affected by impairments in the three mental domains. Finally, we provide suggestions for improving learningbased interventions to prevent and treat ODD and CD.

In the neurobiological overview, we primarily focus on studies including clinical samples of children and adolescents with ODD and CD, and children and adolescents with these disorders and/or psychopathic or callous-unemotional (CU) traits, that is, the affective factor of psychopathy (Frick and White 2008). The samples of many studies consist of both children and adolescents with ODD and children and adolescents with $\mathrm{CD}$, here referred to as disruptive behavior disorders (DBDs). In order to better understand the development of ODD and CD, we also discuss several subclinical community studies that classify subject groups on the basis of aggressive behavior, antisocial behavior, and delinquent behavior.

\section{Punishment Processing}

Young children learn to make associations between inappropriate behaviors and (threats of) punishment. Yet, children need to be sensitive to punishment cues in order to learn refraining from inappropriate behaviors. Learning to refrain from inappropriate behaviors is indeed based on classical (aversive) conditioning. For example, aversive conditioning involves learning to associate hitting another child with subsequent punishment or the perception of the distress of the victim. Lack of fear in children can explain poor socialization because low fear of punishment would reduce the effectiveness of classical conditioning (Lykken 1957). Aversive conditioning is crucial for children as it results in anticipatory fear whenever children consider behaving inappropriately as well as in discomfort (e.g., guilt and remorse) occasioned by committed antisocial behavior (Kochanska 1993). The neurobiological system involved in punishment processing consists of the amygdala, the sympathic nervous system, and the hypothalamus-pituitary-adrenal axis. The amygdala has been widely implicated in learning and expressing the association of 
certain undesirable behaviors with punishment, as shown by studies on classical (fear) conditioning and operant conditioning (e.g., passive avoidance learning) (Davis and Whalen 2001; Cardinal et al. 2002; Maren and Quirk 2004; Balleine and Killcross 2006; Phelps and Ledoux 2005). Thus, impaired functioning of the amygdala associated with decreased aversive stimulus-reinforcement associations is thought to be the characteristic of psychopathic individuals (Blair 2007). The amygdala has projections to the hypothalamus, midbrain reticular formation, and brainstem, areas that are associated with autonomic responses.

\section{Amygdala}

A number of studies on structure and function of the amygdala have been performed in children and adolescents with $\mathrm{CD}$ or conduct problems with or without psychopathic characteristics. In adolescents with early-onset CD, most of whom were comorbid with attention deficit hyperactivity disorder (ADHD), reduced gray matter volumes were found in a variety of brain regions, including the amygdala relative to healthy controls. Regression analyses indicated that $\mathrm{CD}$ symptoms were primarily correlated with gray matter reductions in temporal lobes, including the amygdala, as well as the prefrontal cortex (Huebner et al. 2008). In addition, reduced gray matter volumes in the left amygdala have been found in adolescents with $\mathrm{CD}$, relative to healthy controls (Sterzer et al. 2007). Likewise, gray matter volume reductions in the bilateral amygdala have been found in adolescents with $\mathrm{CD}$, relative to healthy comparison subjects (Fairchild et al. 2011). In contrast, one study failed to find structural deviances of the amygdala in boys with CU traits (De Brito et al. 2009). Although the boys in this study had conduct problems, the presence of ODD or CD was not assessed, however. Thus, the negative findings could have been due to the presence of a less severe form of psychopathology in these boys.

Findings from a functional magnetic resonance imaging (fMRI) study suggest left amygdala hyporeactivity to negative emotional stimuli in children and adolescents aged 9-15 years with CD (Sterzer et al. 2005). Likewise, in an fMRI study, DBD children and adolescents (aged 10-17 years) with CU traits, many of whom also had comorbid ADHD, were found to have reduced amygdala responsiveness during the presentation of fearful facial expressions in comparison with healthy controls and youth with ADHD. Interestingly, in this study, functional connectivity analyses demonstrated greater correlations between the amygdala and ventromedial prefrontal cortex in healthy controls and youth with ADHD relative to those with DBDs and CU traits (Marsh et al. 2008). In another fMRI study, boys with conduct problems and elevated levels of CU traits who also had ADHD symptoms showed less right amygdala activity to fearful faces compared with healthy controls; these differences remained after controlling for ADHD symptoms (Jones et al. 2009). In summary, these studies reveal evidence of deficits in amygdala function in children and adolescents with DBDs or conduct problems with or without psychopathic or CU traits. The above-mentioned studies all are cross-sectional. Thus, although these studies may help explain the occurrence of ODD and CD symptoms in elementary school children and adolescents, they are not informative about the development of these symptoms in young children.

\section{Sympathetic Nervous System}

The neural circuit network involved in responsiveness to aversive stimuli also comprises the sympathetic branches of the autonomic nervous system. In a prospective study, fear conditioning using electrodermal responsivity was assessed in children at ages 3, 4, 5, 6, and 8. It was shown that poor fear conditioning from ages 3-8 years is associated with aggression at age 8 (Gao et al. 2010a). Furthermore, it appeared that poor fear conditioning at age 3 predisposes to crime at age 23 (Gao et al. 2010b).

Besides this important series of studies that demonstrate poor fear conditioning in young children as a risk factor for developing aggressive and criminal behavior, psychopathyprone adolescent boys, relative to healthy controls, have also been found to display reduced sympathetic electrodermal activity in anticipation of and in response to an aversive stimulus (Fung et al. 2005). Importantly, no differences were found between antisocial non-psychopathic boys and antisocial psychopathy-prone boys. Thus, the antisocial component of psychopathy may be associated with electrodermal hyporesponsivity. In another study, children and adolescents with CD comorbid with ADHD showed low skin conductance responses to aversive stimuli as well as to (positive and negative) emotional stimuli and to neutral pictures, compared to children and adolescents with ADHD-only and healthy controls (Herpertz et al. 2005). The authors concluded that this general autonomic hyporeactivity may reflect a deficit in associative information-processing systems that normally produce adaptive cognitive-emotional reactions.

\section{Hypothalamus-Pituitary-Adrenal Axis}

The neural circuit network involved in responsiveness to aversive stimuli and stress also comprises the hypothalamus-pituitary-adrenal axis (LeDoux 2002). Cortisol secretion by the adrenal cortex is controlled by adrenocorticotropic hormone released from the pituitary, which is regulated by corticotrophin-releasing hormone from the 
hypothalamus. Corticotrophin-releasing hormone is released in response to stress and subsequent activation of the amygdala and prefrontal cortex. Reduced cortisol reactivity to stress has been found in children with DBDs, many of whom had comorbid ADHD (van Goozen et al. 1998, 2000). Importantly, low cortisol responsivity during stress appears to be specific to DBDs. In a study that examined children with DBDs, children with ADHD, and healthy controls, only children with DBDs showed a blunted cortisol response (Snoek et al. 2004).

\section{Neurochemistry}

On the neurochemical level, studies examining serotonergic (5-HT) and noradrenergic neurotransmission in the central nervous system are particularly relevant. 5-HT neurotransmission has, among other functions, been implicated in the sensitivity to punishment and aversive signals (Cools et al. 2008). There is support for an inverse relationship between 5-HT measures and aggressive behavior in children and adolescents with ODD and CD, although this relationship is less clear than in adults (for an extensive review, see Matthys et al. 2012). Noradrenergic neurotransmission may also play an important role in behavioral arousal associated with punishment (Berridge and Waterhouse 2003). That is, if signals associated with punishment do not lead to the noradrenergically driven increase of attention and change in emotional state, these signals become less meaningful. There is some evidence to suggest decreased noradrenergic functioning in the DBDs (for an extensive review, see Matthys et al. 2012).

\section{Summary}

Studies on amygdala function, electrodermal fear conditioning, cortisol reactivity to stress, and serotonergic and noradrenergic neurotransmission indicate that reduced sensitivity to punishment and aversive cues plays a role in ODD and CD symptoms. Children and adolescents with ODD and CD thus may have difficulties in learning to refrain from inappropriate behaviors.

\section{Reward Processing}

In young children, newly acquired behaviors are likely to become part of their behavioral repertoire when these behaviors are rewarded. Indeed, according to operant conditioning principles, behaviors that are rewarded are more likely to be repeated. In learning new behaviors, classical conditioning is also thought to be involved, that is, in making the association between behaviors and rewarding stimuli. Thus, low sensitivity to reward will reduce the learning of appropriate behaviors. Besides, low reward sensitivity may also result in reduced motivation to obtain natural rewards. The associated unpleasant effect may incite children and adolescents to sensation-seeking, which may manifest in antisocial behavior and in searching unnaturally strong rewards such as drugs of abuse. The neurobiological system involved in reward processing consists of the amygdala, the orbitofrontal cortex, and the striatum (Cardinal et al. 2002; Balleine and Killcross 2006; Blair 2007; Schoenbaum and Roesch 2005). The amygdala is thought to subserve the formation of stimulus-outcome associations based on reward, and the amygdala closely interacts with the orbitofrontal cortex, which has been implicated in the generation of reinforcement expectations. The orbitofrontal cortex and striatum also play a role in error prediction, thus facilitating learning of reward (O’Doherty et al. 2006).

\section{Amygdala, Orbitofrontal Cortex, and Striatum}

Reduced gray matter volumes in the amygdala in adolescents with CD discussed above (Fairchild et al. 2011; Huebner et al. 2008; Sterzer et al. 2007) may result in altered reward processing. With respect to the functioning of the amygdala, the orbitofrontal cortex and the caudate nucleus (i.e., part of the dorsal striatum), an fMRI study using a passive avoidance task in adolescents with DBDs and psychopathic traits is relevant here (Finger et al. 2011). In this task, participants learned to respond to stimuli that engender reward and to refrain from responding to stimuli that engender punishment. First, amygdala responsiveness throughout the learning task was lower in adolescents with DBDs than in controls associated with an impairment in making stimulus-reinforcement associations. Second, while performing the task, less orbitofrontal and caudate nucleus responsiveness to early exposure in the task was shown in adolescents with DBDs relative to comparison youths, resulting in disrupted prediction error signaling. Third, less orbitofrontal responsiveness to rewards was shown, associated with decreased representation of reward expectancy values. Thus, the integrated functioning of the amygdala, caudate nucleus, and orbitofrontal cortex seems to be disrupted in adolescents with DBDs and psychopathic traits. More evidence for orbitofrontal dysfunction in CD comes from an fMRI study that assessed brain activation during a continuous performance task measuring sustained attention and the effects of reward on performance. Children and adolescents with CD without ADHD showed underactivation in the right orbitofrontal cortex during the reward condition relative to healthy comparison subjects and children and adolescents with ADHD without CD (Rubia et al. 2009a). These studies suggest that compromised processing of reward cues may result in impaired reinforcement processing. 


\section{Heart Rate}

Heart rate has been linked to reward processing (Fowles 1980) whereby low resting heart rate may reflect reduced reactivity to rewarding stimuli associated with an unpleasant state. In support of this interpretation, preschool boys who chose to watch videotapes of intense anger had lower basal heart rates than boys who chose to watch videotapes of mild anger. Thus, boys with a lower heart rate level might have sought a higher level of stimulation to experience a pleasant emotional state (El-Sheikh et al. 1994). A longitudinal general population study found that sensation-seeking, but not behavioral disinhibition, in boys at age 13.5 and 16 mediated the relationship between low resting heart rate at age 11 and rule breaking at age 16 (Sijtsema et al. 2010). This study supports sensationseeking theory, which states that rule breaking in adolescence serves to alleviate the unpleasant state of stimulus hyporeactivity associated with low resting heart rate, to experience a pleasant level of emotional stimulation.

\section{Neurochemistry}

Dopamine is an important neurotransmitter involved in several aspects of reward processing. For example, dopamine has been implicated in reward-related learning processes, reward expectation, incentive salience, and incentive motivation (Berridge 2007; Bromberg-Martin et al. 2010; Cardinal et al. 2002; Kelley 2004; Robbins and Everitt 2007; Schultz 2007). Lower activity of brain dopamine systems could therefore result in reduced salience of positive emotional stimuli in the environment, lack of motivation to exert effort to obtain rewards, impaired learning of stimulus-reward associations or reduced influence of reward-associated stimuli on positive emotions. Both genetic studies (e.g., of catechol-O-methyltransferase; COMT) and studies of the metabolite of dopamine suggest decreased dopaminergic functioning in ODD and CD (for an extensive review, see Matthys et al. 2012). Furthermore, studies of the effectiveness of psychostimulants, which enhance dopaminergic neurotransmission by blocking the reuptake and/or enhancing the release of dopamine (Fone and Nutt 2005), may give insight into the neurochemical mechanisms of ODD and CD. These studies have been conducted primarily in ADHD, but in view of the substantial comorbidity of the DBDs with ADHD (Angold et al. 1999), it is no surprise that many studies have investigated the effect of psychostimulant drugs, such as methylphenidate and amphetamine, in subjects with ADHD with or without DBD or DBD symptoms. The effect of psychostimulants on disruptive behaviors has been shown convincingly in a number of studies (Ghuman et al., 2007; Greenhill et al. 2006; Klein et al. 1997; Swanson et al. 2001; Taylor et al. 1987) and in one meta-analysis (Connor et al. 2002); in the latter study, the overall weighted mean effect size was 0.84 for overt and 0.69 for covert aggression-related behaviors in ADHD. Together, these studies suggest that improved dopaminergic function results in a reduction of ODD and CD symptoms.

With respect to the behavioral mechanism of action of psychostimulants, methylphenidate has been shown to exert positive effects on cognitive performance by increasing task salience (Volkow et al. 2004). As incentive salience or "wanting" is particularly influenced by dopaminergic neurotransmission (Berridge 2007), an increase in incentive salience by psychostimulants might explain why parents of children and adolescents with DBDs treated with methylphenidate report improvement in their children's attention, motivation to comply, and to engage more positively in social interactions. Indeed, evidence from animal studies indicates that treatment with psychostimulant drugs enhances the influence of salient, reward-, or punishmentassociated cues on behavior (Robbins et al. 1983; Killcross et al. 1997; Wyvell and Berridge 2000) and can also enhance reward- and punishment-driven learning processes (Carr and White 1984; Hitchcott et al. 1997; Phillips et al. 2003; Tye et al. 2010). Finally, an increase in dopaminergic functioning by psychostimulants may also facilitate appropriate reward expectations (Berridge 2007; Bromberg-Martin et al. 2010; Cardinal et al. 2002; Kelley 2004; Robbins and Everitt 2007; Schultz 2007).

\section{Summary}

Studies on the amygdala, orbitofrontal cortex, and caudate nucleus, on heart rate, and on dopaminergic functioning indicate that reduced reward sensitivity plays a role in ODD and CD symptoms. Children and adolescents with ODD and CD may therefore have difficulties in learning appropriate behaviors.

\section{Cognitive Control}

In everyday situations, children are continuously faced with problems they need to solve. Various cognitive control or executive functions are involved in problem solving, such as planning, working memory, inhibition of inappropriate responses, flexibility in adaptation to environmental changes, and decision making (Nigg 2006). These functions are subserved by the frontal cortex. Specifically, the paralimbic system comprising orbitofrontal, superior temporal, cingulate cortices, and limbic brain regions mediates the cognitive control of emotion and motivation (Blair 2004). Due to maturation of the frontal cortex with age (Durston et al. 2001), cognitive control over behavior increases in children and adolescents. 


\section{Executive Functions}

According to a meta-analysis of studies in school-aged children, adolescents, and adults with externalizing disorders, the average mean effect size (Cohen's d) of a variety of executive measures for the groups with antisocial behavior (criminality, delinquency, CD, psychopathy, antisocial personality disorder) was 0.62 , whereas the effect size for $\mathrm{CD}$ was 0.36 (Morgan and Lilienfeld 2000). However, the possible role of ADHD comorbidity in executive functions impairments in $\mathrm{CD}$ was not examined in this study, while another meta-analysis revealed that deficits in executive functions in $\mathrm{CD}$ are likely due to the presence of comorbid ADHD (Pennington and Ozonoff 1996). On the other hand, it was shown in a further meta-analysis that deficits in response inhibition are not only found in elementary school children with ADHD, but also in children with DBD without comorbid ADHD (Oosterlaan et al. 1998). Likewise, impairments in inhibition have been found in preschool children with aggressive behavior when compared with non-aggressive preschoolers, and these impairments were maintained after controlling for attention problems (Raaijmakers et al. 2008). Impairments in inhibition were also found in preschool children with DBDs without ADHD comorbidity relative to normal controls (Schoemaker et al. 2012). Impaired executive functioning capacities such as planning ability and inhibitory control have been linked specifically to reactive aggression (Ellis et al. 2009).

Results of studies in ODD and CD are even more consistent when motivational factors are involved in executive functions. In this respect, the distinction has been made between "cool" executive functions and "hot" executive functions that involve reward and punishment (Rubia 2011). For example, the tendency to continue a previously rewarded response that is now punished, that is, response perseveration, has been demonstrated in children and adolescents with DBDs (Daugherty and Quay 1991; Matthys et al. 1998, 2004; van Goozen et al. 2004). Response perseveration may contribute to impairments in decision making rendering individuals unable to shift their behavior away from immediate rewards that are associated with even larger punishments. Disadvantageous decision making indicating favoring immediate rewards despite long-term punishments has been shown in children and adolescents with psychopathic tendencies (Blair et al. 2001), adolescents with ADHD or CD (Ernst et al. 2003), children with ODD most of whom were comorbid with ADHD (Luman et al. 2010), adolescents with CD some of who had comorbid ADHD (Fairchild et al. 2009), and adolescents with both DBDs and substance dependence (Schutter et al. 2011).

\section{Paralimbic System}

A number of studies on structure and function of the paralimbic system have been performed in children and adolescents with ODD and CD or conduct problems with or without psychopathic characteristics (for extensive reviews, see Rubia 2011, and Matthys et al. 2012). For example, in a study with early-onset adolescents with CD (most of whom were comorbid with ADHD), whole brain volume analysis showed reduced gray matter volumes in the left orbitofrontal region, bilaterally in the temporal lobes, and in the amygdala and hippocampus on the left side compared with healthy controls. Mean total gray matter volume was $6 \%$ smaller in the clinical group. Regression analyses indicated that CD symptoms were correlated primarily with gray matter reductions in limbic brain structures including the amygdala and the prefrontal areas, whereas hyperactive/impulsive symptoms were correlated with gray matter abnormalities in the frontoparietal and temporal cortices (Huebner et al. 2008).

With respect to the functioning of the prefrontal cortex, children and adolescents with CD and children and adolescents with ADHD performing an inhibition task showed reduced activation in the posterior cingulate compared to healthy controls in an fMRI study. Children and adolescents with $\mathrm{CD}$ showed reduced activation in temporal-parietal regions during failed inhibition when compared with the other groups. Since participants obtained feedback about their inhibition failures, the results suggest that performance monitoring networks are dysfunctional in CD when compared to ADHD and healthy controls (Rubia et al. 2008). Consistent with this notion is the observation that adolescents with psychopathic traits and ODD or CD, the majority of whom also had comorbid ADHD, showed abnormal responses of the ventromedial prefrontal cortex during punished errors in a reversal learning task, as compared to adolescents with ADHD and healthy controls (Finger et al. 2008a, b). According to Rubia et al. (2008), this could mean that adolescents with CD care less about their mistakes than adolescents with ADHD and healthy controls, which is in line with evidence that children with DBDs are undermotivated and respond less to negative feedback than controls (Matthys et al. 2004; van Goozen et al. 2004).

As already discussed in the section on reward processing, lower responsiveness to reward outcome information within the orbitofrontal cortex has been shown in children and adolescents with CD (Rubia et al. 2009a, b). Blair (2004) has argued that orbitofrontal cortex dysfunctioning is involved in the modulation of reactive aggression. Executive functioning capacities such as planning ability and inhibitory control have been specifically linked to reactive aggression (Ellis et al. 2009). As the orbitofrontal cortex is involved in the computations of expectation of reward and violations of expected 
reward result in frustration, orbitofrontal dysfunction as shown in the studies by Finger et al. (2011), and by Rubia et al. (2009a) may indeed be associated with reactive aggression. During interference inhibition and attention allocation, boys with $\mathrm{CD}$ only showed reduced activation in right middle and superior temporal and parietal regions compared to controls. Impaired function of these areas possibly accounts for attentional deficits in CD, causing more errors (Rubia et al. 2009b).

The anterior cingulate cortex, which is involved in emotion processing and social functioning (Bush et al. 2000), has also been studied in children and adolescents with DBDs. In an fMRI study by Stadler et al. (2007), children aged 9-15 years with CD, the majority of who also had comorbid ADHD, viewed negative pictures, and showed reduced activation in the dorsal part of the anterior cingulate cortex, that is, the part believed to be involved in the cognitive control of emotional behavior, as compared to healthy controls. This abnormal suppression of neural activity may result in a failure to cognitively control emotional behavior. Similarly, abnormal right anterior cingulate cortex activation during the presentation of images with negative valence was shown in children and adolescents aged 9-15 years with CD most of whom also had comorbid ADHD relative to healthy controls (Sterzer et al. 2005). Impaired functioning of the anterior cingulate cortex associated with the cognitive control of emotions may thus be involved in DBD. Finally, during cognitive flexibility, brain dysfunctions in left superior temporal, bilateral parietal, and occipital regions were observed in CD boys relative to healthy controls (Rubia et al. 2010).

\section{Summary}

Impairments in executive or cognitive control functions have been shown, that is, in inhibition and decision making, especially when motivational factors (reward and punishment) are involved. Likewise, ODD and CD have been associated with structural deficits and impaired functioning of the paralimbic system comprising the orbitofrontal cortex, superior temporal lobes, and cingulate cortices. Children and adolescents with ODD and CD thus are less likely to learn from their mistakes. Consequently, they may not learn to make appropriate decisions in the context of punishment and reward. Impaired attention and cognitive flexibility also have been shown in ODD and CD. Finally, problems in the computations of expectation of reward and violations of expected reward may result in reactive aggression.

Impaired Neurocognitive Functions Affect Social Learning Processes

According to the conceptual framework presented here, adequate functioning of the three mental domains is necessary for the occurrence of appropriate social learning processes. Results of neurobiological studies in ODD and $\mathrm{CD}$ suggest that impaired neurocognitive functions, that is, the functions exerted by the three mental domains, affect the social learning processes in these disorders. First, the ability to make associations between behaviors and positive or negative consequences is compromised. As a result, both learning of appropriate behavior and learning to refrain from inappropriate behavior may be affected. Second, problem solving is impaired due to deficiencies in inhibition, attention, cognitive flexibility, and decision making. As a result, children and adolescents may have difficulty learning to optimize their behavior in changeable environments. Prospective studies, however, are needed to further clarify the role of impaired neurocognitive functions in the development and in the maintenance of ODD and CD. Importantly, it should be excluded that the social learning difficulties in ODD and CD are exclusively due to general deficits of learning.

The conceptualization of the neurobiological systems in terms of functions allows for the inclusion of environmental factors to better understand social learning difficulties in ODD and CD. For example, as children learn to refrain from inappropriate behaviors based on aversive conditioning, difficulties in the processing of punishment cues resulting in fewer associations between inappropriate behaviors and punishment may put children at risk of developing ODD and CD symptoms, especially in families with inconsistent discipline. Similarly, difficulties in the processing of reward cues may result in compromised learning of appropriate behaviors. This is an important issue that has often been overlooked and that may help answer the question of why some children with ODD and $\mathrm{CD}$ do not learn to substitute inappropriate behaviors with appropriate behaviors, as children typically do. To more fully understand this social learning difficulty, we need to account for the parenting characteristics in the families of these children, such as the amount and quality of praising children's appropriate behaviors. Finally, decreased cognitive control in children and adolescents may result in reactive aggression and risk-taking behavior, maybe especially when parents of these children and adolescents do not present appropriate models of coping in situations of frustration because of difficulties in self-control themselves.

Improving the Tailoring of Psychological Treatments

The role of impaired neurocognitive functions in the social learning processes of ODD and CD has consequences for the deployment of interventions with specific children. Over the years, behavioral and cognitive-behavioral interventions based on social learning have been developed, 
adapted, and tested. On the one hand, there is evidence of effectiveness of interventions in ODD and CD: the mean effect size of behavioral parent training is 0.47 (range from 1.68 to -0.06$)$ and the mean effect size of cognitive behavior therapy is 0.35 (range from 1.87 to -1.04) (McCart et al. 2006). These mean effect sizes, however, are not large. Furthermore, these effect sizes are based on studies conducted in highly controlled research settings in which, for example, therapists are specifically trained to conduct the intervention and treatment fidelity is checked. Dissemination research does suggest that these interventions can be implemented effectively in real-world settings when intensive training is provided to clinicians (Lochman et al. 2009) but it remains unclear whether the effect sizes obtained in everyday clinical practice would be as large as evident in the clinical trials.

One may question why, despite intense efforts for five decades to develop behavior parent training programs and for four decades to develop cognitive behavior therapy programs, effects of these interventions still are small to moderate, at best. Based on the evidence of impaired neurocognitive functions in ODD and CD, limited effectiveness of these learning-based interventions may be caused by difficulties in social learning by children and adolescents with ODD and CD. The neurocognitive characteristics mentioned above, resulting in difficulty to refrain from inappropriate behaviors due to low punishment sensitivity, however, refer to characteristics that have been found in groups of children and adolescents with ODD and CD. This also applies to difficulties in learning to behave appropriately due to low reward sensitivity and to difficulties in learning to optimize behavior in changeable environments due to impaired cognitive control of behavior and emotions. Thus, at an individual level children and adolescents with ODD or CD may differ in these neurocognitive characteristics and associated learning difficulties, as ODD and $\mathrm{CD}$ are heterogeneous disorders depending on, i.a., comorbidity with ADHD, association with CU traits and anxiety symptoms. Importantly, recent research suggests that ODD is a separate disorder to be distinguished from CD both in symptomatology, comorbidity, and development (Rowe et al. 2010; Nock et al. 2007; Stringaris and Goodman 2009a, b). Indeed, with respect to ODD symptomatology, a defiant/headstrong behavior cluster (argues with adults, defies adults' requests, deliberately annoys people, blames others) can be differentiated from an irritability cluster (temper tantrums, touchy or easily annoyed, angry, and resentful) (Stringaris and Goodman 2009a; Rowe et al. 2010). ODD may therefore be considered a mixed disorder of behavior and emotion. The defiant/headstrong behavior cluster is associated specifically with the development of $\mathrm{CD}$, while the irritability factor is associated specifically with the development of anxiety and mood disorders (Stringaris and Goodman 2009b). Thus, the neurocognitive characteristics and associated social learning difficulties in ODD may in part differ from those in $\mathrm{CD}$, and even among $\mathrm{CD}$ children and ODD children, there are likely to be variations in the degree of neurocognitive difficulties evident.

We here suggest that for clinical purposes, the neurocognitive characteristics in children and adolescents with ODD and CD need to be assessed individually. This clinical assessment may consist of a mixture of assessment procedures such as standardized behavioral observation of response to reward and punishment cues, neuropsychological tests of responsivity to reward and punishment, neuropsychological tests of executive functions, and judgements of children's and adolescents' neurocognitive functioning by parents and teachers. Instead of delivering intervention programs in a standardized way, these programs should be individualized depending on the weaknesses and strengths of the neurocognitive characteristics of the child and the adolescent associated with their social learning difficulties. We now discuss this individualization of psychological interventions indicating how the three mental domains could relate to intervention elements.

Evidence-based interventions for children with ODD and CD often include behavioral parent training (BPT) with parents, school-based behavioral consultation with teachers, and cognitive-behavioral intervention (CBI) with the children. CBI often becomes an increasingly important intervention component from middle childhood through adolescence, while BPT is important at all ages. Typical, or common, elements of evidence-based interventions for children with ODD and CD have been described in recent years (e.g., Matthys and Lochman 2010). Evidence-based intervention work with parents in the home context, and also with teachers and other personnel in the school context, typically includes: (1) increase of positive attention, social reinforcement, and rewards, (2) enhanced antecedent control through clarification of instructions and commands, (3) improved consistency of clearly defined (and non-harsh) discipline methods, and (4) improved monitoring and supervision of children's behavior. Both BPT and CBI often include a focus on (5) enhanced stress management and emotional regulation (anger management) skills. Other typical elements of CBI with children include: (6) enhanced goal setting and behavioral goal completion abilities, (7) enhanced perspective taking and abilities to accurately perceive the intentions of others, (8) improved social problemsolving skills, and (9) improved social skills and abilities to become more engaged with less-deviant peer groups. This listing of typical common elements is not totally comprehensive but does address primary targets of intervention.

So, while all of these domains are conceptualized as key mechanisms of action in intervention with ODD and CD 
children, how would relative emphasis on these typical common elements shift if children had difficulties in a specific mental domain? If a child had relative deficits in the mental domain of reward processing, the first two of the common elements noted, in work with parents and teachers, would be of special value. Thus, more time would be spent with parents and teachers than would typically be the case on parent-child special time, use of praise and point systems with rewards, and focus on how clearly stated instructions to children is more likely to lead to compliant behaviors and then to positive reinforcement. Importantly, BPT and behavioral school consultation in general assist parents and teachers to relabel inappropriate behaviors such as fighting with siblings or defiance toward parents into "positive opposites" (Kazdin 2005) or "prosocial opposites" (Wells et al. 2008) such as cooperative play with siblings and compliance toward parents. Adults, then, learn how to use labeled praise appropriately (e.g., having eye contact with the child, praising the child with enthusiasm, and describing the particular behavior that is praised; Webster-Stratton 2001; Wells et al. 2008).

Individualization of use of rewards and praising may help increase the efficiency of these parenting skills; for example, if it is demonstrated that the processing of reward cues in a child with ODD/CD comorbid with ADHD is severely impaired, then parents and teachers can practice getting the attention of the child by touching and holding the child's attention by using eye contact. Likewise, if the emotional significance of the positive message of praise is less well processed, both verbal and nonverbal enthusiasm would be particular relevant. In children with borderline intelligence and deficient reward processing, adults need to be extremely clear in specifying the behavior that is expected for the child to understand what behavior is being praised. Enhanced focus on rewards within the CBI would also be useful for children with deficient reward processing. To increase children's attention to rewards, shorter behavioral monitoring intervals should be used during intervention sessions and in children's weekly behavioral goals in school or home settings, and more frequent and more salient rewards should be used as consequences for children accomplishing behavioral goals during intervention and in real-world class and home settings (e.g., Lochman et al. 2008).

With regard to the mental domain of punishment processing, relatively greater emphasis in work with parents and teachers can be placed on clarifying antecedent control and monitoring of children, and use of more consistent and less harsh discipline methods (e.g., withdrawal of privileges, work chores, and time-out) (typical elements 2-4 above). It is clear that punishment as the presentation of an aversive stimulus such as slapping is inappropriate while the removal of a positive stimulus such as losing privileges may be adequate when used selectively. Because children and adolescents with deficient punishment processing characteristics probably are at risk for escalating cycles of punishment as milder forms of punishment may not "work" for them (see also Dadds and Salmon 2003), it is also important to emphasize the first typical element above by relabeling inappropriate behaviors into positive opposites. It is particularly critical to positively reinforce the appropriate behaviors using praise and token economy rewards for these children and adolescents. In CBI work with children with deficient punishment processing, it is especially important to assist children to experience clear consequences for their behaviors and to more accurately expect negative outcomes, or consequences, for their behavior. CBI elements that appear to be especially critical for enhancing children's outcome expectations for their behaviors are short- and long-term goal setting activities (typical element 6 above) and a highly detailed and comprehensive approach to considering consequences for possible problem solutions during social problem-solving activities (typical element 8 above).

While children with a weak cognitive control domain can benefit from the BPT typical elements (1-4), some of the CBI activities are likely to be particularly relevant. Failures to inhibit one's behavior appropriately can be associated with poor self and emotional regulation skills. Thus, CBI elements designed to assist with emotional regulation could be an area of particular emphasis. As children develop better perspective taking skills (typical element 7 above), they can be less prone to impulsively and erroneously misinterpret the intentions of others (thus reducing hostile attributional biases). Children's development of improved anger management skills (typical element 5 above) and deliberate social problem-solving skills ("stop-think-what should I do?" typical element 8 above) can also assist children's inhibitory abilities, and these typical elements can thus receive greater emphasis with these children. Decision making is a key aspect of one of the last steps of the problem-solving model and can be an important area of emphasis for children with cognitive control problems. Addressing children's expectations of long-term consequences and outcomes of behaviors can assist children to reduce inappropriate decision making, which favors immediate rewards despite long-term punishments and results in risky decisions.

In conclusion, in the etiology of ODD and CD, more attention should be paid to neurobiological factors involved in the social learning processes that play a role in the initiation and maintenance of these disorders. Likewise, the assessment of the neurocognitive characteristics associated with the social learning processes may help individualize interventions. As a result, the effectiveness of learningbased interventions may increase. 


\section{References}

American Psychiatric Association (2000). Diagnostic and statistical manual of mental disorders, 4th ed., Text revision (DSM-IV-TR). Washington DC: American Psychiatric Association.

Angold, A., Costello, J. E., \& Erkanli, A. (1999). Comorbidity. Journal of Child Psychology and Psychiatry, 40, 57-87.

Balleine, B. W., \& Killcross, S. (2006). Parallel incentive processing: An integral view of amygdala functioning. Trends in Neurosciences, 29, 272-279.

Bandura, A. (1973). Aggression: A social learning analysis. Englewood Cliffs, NJ: Prentice-Hall.

Berridge, K. C. (2007). The debate over dopamine's role in reward: The case for incentive salience. Psychopharmacology (Berlin), 191, 391-431.

Berridge, C. W., \& Waterhouse, B. D. (2003). The locus coeruleusnoradrenergic system: Modulation of behavioral state and statedependent cognitive processes. Brain Research Reviews, 42, 33-84.

Blair, R. J. R. (2004). The roles of orbital frontal cortex in the modulation of antisocial behaviour. Brain and Cognition, 55, 198-208.

Blair, R. J. R. (2007). Dysfunctions of medial and lateral orbitofrontal cortex in psychopathy. Annals of the New York Academy of Sciences, 1121, 461-479.

Blair, R. J. R., Colledge, E., \& Mitchell, D. G. V. (2001). Somatic markers and response reversal: Is there orbitofrontal cortex dysfunction in boys with psychopathic tendencies? Journal of Abnormal Child Psychology, 29, 499-511.

Bromberg-Martin, E. S., Matsumoto, M., \& Hikosaka, O. (2010). Dopamine in motivational control: Rewarding, aversive, and alerting. Neuron, 68, 815-834.

Bush, G., Luu, P., \& Posner, M. I. (2000). Cognitive and emotional influences in anterior cingulated cortex. Trends in Cognitive Science, 4, 215-222.

Carr, G. D., \& White, N. M. (1984). The relationship between stereotypy and memory improvement produced by amphetamine. Psychopharmacology, 82, 203-209.

Cardinal, R. N., Parkinson, J. A., Hall, J., \& Everitt, B. J. (2002). Emotion and motivation: The role of the amygdala, ventral striatum, and prefrontal cortex. Neuroscience and Biobehavioral Reviews, 26, 321-352.

Connor, D. F., Glatt, S., Lopez, I., Jackson, D., \& Melloni, R. (2002). Psychopharmacology and Aggression. I A Meta analysis of stimulant effects on overt-covert aggression-related behaviors in ADHD. Journal of the American Academy of Child and Adolescent Psychiatry, 41, 253-261.

Cools, R., Roberts, A. C., \& Robbins, T. W. (2008). Serotoninergic regulation of emotional and behavioural control processes. Trends in Cognitive Sciences, 12, 31-40.

Dadds, M. R., \& Salmon, K. (2003). Punishment insensitivity and parenting: Temperament and learning as interacting risks for antisocial behaviour. Clinical Child and Family Psychology Review, 6, 69-86.

Daugherty, T. K., \& Quay, H. C. (1991). Response perseveration and delayed responding in childhood behavior disorders. Journal of Child Psychology and Psychiatry, 32, 453-461.

Davis, M., \& Whalen, P. J. (2001). The amygdala: Vigilance and emotion. Molecular Psychiatry, 6, 13-34.

De Brito, S. A., Mechelli, A., Wilke, M., Laurens, K. R., Jones, A. P., Barker, G. J., et al. (2009). Size matters: Increased grey matter in boys with conduct problems and callous-unemotional traits. Brain, 132, 843-852.

Durston, S., Hulshoff Pol, H. E., Casey, B. J., Giedd, J. N., Buitelaar, J. K., \& van Engeland, H. (2001). Anatomical MRI of the developing human brain: What have we learned? Journal of the American Academy of Child and Adolescent Psychiatry, 40, 2149-2157.

Ellis, M. L., Weiss, B., \& Lochman, J. E. (2009). Executive functions in children: Associations with aggressive behavior and social appraisal processing. Journal of Abnormal Child Psychology, 37, 945-956.

El-Sheikh, M., Ballard, M., \& Cummings, E. M. (1994). Individual differences in preschoolers' physiological and verbal responses to videotaped angry interactions. Journal of Abnormal Child Psychology, 22, 303-320.

Ernst, M., \& Fudge, J. L. (2009). A developmental neurobiological model of motivated behavior: Anatomy, connectivity and ontogeny of the triadic nodes. Neuroscience and Biobehavioral Reviews, 33, 367-382.

Ernst, M., Grant, S. J., London, E. D., Contoreggi, C. S., Kimes, A. S., \& Spurgeon, L. (2003). Decision making in adolescents with behaviour disorders and adults with substance abuse. American Journal of Psychiatry, 160, 33-40.

Fairchild, G., Passamonti, L., Hurford, G., Hagan, C. C., von dem Hagen, E. A. H., van Goozen, S. H. M., et al. (2011). Brain structure abnormalities in early-onset and adolescent-onset conduct disorder. American Journal of Psychiatry, 168, 624-633.

Fairchild, G., van Goozen, S. H. M., Stollery, S. J., Aitken, M. R. F., Savage, J., Moore, S. C., et al. (2009). Decision making and executive function in male adolescents with early-onset or adolescence-onset conduct disorder and control subjects. Biological Psychiatry, 66, 162-168.

Finger, E. C., Marsh, A. A., Blair, K. S., Reid, M. E., Sims, C., Ng, P., et al. (2008a). Disrupted reinforcement signalling in the orbitofrontal cortex and caudate in youths with conduct disorder or oppositional defiant disorder and a high level of psychopathic traits. American Journal of Psychiatry, 168, 152-162.

Finger, E. C., Marsh, A. A., Mitchell, D. G., Reid, M. E., Sims, C., Budhani, S., et al. (2008b). Abnormal ventromedial prefrontal cortex function in children with psychopathic traits during reversal learning. Archives of General Psychiatry, 65, 586-594.

Finger, E. C., Marsh, A. A., Blair, K. S., Reid, M. E., Sims, C., Ng, P., et al. (2011). Disrupted reinforcement signaling in the orbitofrontal cortex and caudate in youths with conduct disorder or oppositional defiant disorder and a high level of psychopathic traits. American Journal of Psychiatry, 168, 152-162.

Fone, K. C., \& Nutt, D. J. (2005). Stimulants: use and abuse in the treatment of attention deficit hyperactivity disorder. Current Opinion in Pharmacology, 5, 87-93.

Fowles, D. C. (1980). The three arousal model: Implications of Gray's two-factor learning theory for heart rate, electrodermal activity, and psychopathy. Psychophysiology, 17, 87-104.

Frick, P. J., \& White, S. F. (2008). Research review: The importance of callous-unemotional traits for developmental models of aggressive and antisocial behavior. Journal of Child Psychology and Psychiatry, 49, 359-375.

Fung, M. T., Raine, A., Loeber, R., Lynam, D. R., Steinhauer, S. R., Venables, P. H., et al. (2005). Reduced electrodermal activity in psychopathy-prone adolescents. Journal of Abnormal Psychology, 114, 187-196.

Gao, Y., Raine, A., Venables, P. H., Dawson, M. E., \& Mednick, S. A. (2010a). Reduced electrodermal fear conditioning from ages 3 to 8 years is associated with aggressive behaviour at age 8 years. Journal of Child Psychology and Psychiatry, 51, $550-558$

Gao, Y., Raine, A., Venables, P. H., Dawson, M. E., \& Mednick, S. A. (2010b). Association of poor childhood fear conditioning and adult crime. American Journal of Psychiatry, 167, 56-60.

Ghuman, J. K., Riddle, M. A., Vitiello, B., Greenhill, L. L., Chuang, S. Z., Wigal, S. B., et al. (2007). Comorbidity moderates 
response to methylphenidate in the Preschoolers AttentionDeficit/Hyperactivity Disorder Treatment Study (PATS). Journal of Child and Adolescent Psychopharmacology, 17, 563-579.

Greenhill, L., Kollins, S., Abikoff, H., McCracken, J., Riddle, M., Swanson, J., et al. (2006). Efficacy and safety of immediaterelease methylphenidate treatment for preschoolers with ADHD. Journal of the American Academy of Child and Adolescent Psychiatry, 45, 1284-1293.

Herpertz, S. C., Mueller, B., Qunaibi, M., Lichterveld, C., Konrad, K., \& Herpertz-Dahlmann, B. (2005). Response to emotional stimuli in boys with conduct disorder. American Journal of Psychiatry, $162,1100-1107$.

Hitchcott, P. K., Harmer, C. J., \& Phillips, G. D. (1997). Enhanced acquisition of discriminative approach following intra-amygdala d-amphetamine. Psychopharmacology (Berlin), 132, 237-246.

Huebner, T., Vloet, T. D., Marx, I., Konrad, K., Fink, G. R., Herpertz, S. C., et al. (2008). Morphometric brain abnormalities in boys with conduct disorder. Journal of the American Academy of Child and Adolescent Psychiatry, 47, 540-547.

Jones, A. P., Laurens, K. R., Herba, C. J., \& Viding, E. (2009). Amygdala hypoactivity to fearful faces in boys with conduct problems and callous-unemotional traits. American Journal of Psychiatry, 166, 95-102.

Kazdin, A. E. (2005). Parent management training. Treatment for oppositional, aggressive, and antisocial behaviour in children and adolescents. New York: Oxford University Press.

Kelley, A. E. (2004). Ventral striatal control of appetitive motivation: Role in ingestive behaviour and reward-related learning. Neuroscience and Biobehavioral Reviews, 27, 765-776.

Killcross, A. S., Everitt, B. J., \& Robbins, T. W. (1997). Symmetrical effects of amphetamine and alpha-flupenthixol on conditioned punishment and conditioned reinforcement: Contrasts with midazolam. Psychopharmacology (Berlin), 129, 141-152.

Kim-Cohen, J., Caspi, A., Moffitt, T. E., Harrington, H., Milne, B. J., \& Poulton, R. (2003). Prior juvenile diagnoses in adults with mental disorder: Developmental follow-back of a prospective-longitudinal cohort. Archives of General Psychiatry, 60, 709-717.

Klein, R. G., Abikoff, H., Klass, E., Ganales, D., Seese, L. M., \& Pollack, S. (1997). Clinical efficacy of methylphenidate in conduct disorder with and without attention deficit hyperactivity disorder. Archives of General Psychiatry, 54, 1073-1080.

Kochanska, G. (1993). Toward a synthesis of parental socialization and child development in early development of conscience. Child Development, 64, 325-347.

Lahey, B. B., Miller, T. L., Gordon, R. A., \& Riley, A. W. (1999). Developmental epidemiology of the disruptive behavior disorders. In H. C. Quay \& A. E. Hogan (Eds.), Handbook of disruptive behaviour disorders (pp. 23-48). New York: Kluwer Academic/Plenum Publishers.

LeDoux, J. E. (2002). Synaptic self: How our brains become who we are. New York: Viking.

Lochman, J. E., Boxmeyer, C., Powell, N., Qu, L., Wells, K., \& Windle, M. (2009). Dissemination of the coping power program: Importance of intensity of counselor training. Journal of Consulting and Clinical Psychology, 77, 397-409.

Lochman, J. E., \& Wells, K. C. (2002). Contextual social-cognitive mediators and child outcome: A test of the theoretical model in the Coping Power Program. Development and Psychopathology, 14, 971-993.

Lochman, J. E., Wells, K. C., \& Lenhart, L. A. (2008). Coping Power child group program: Facilitator guide. New York, NY: Oxford.

Luman, M., Sergeant, J. S., Knol, D., \& Oosterlaan, J. (2010). Impaired decision making in oppositional defiant disorder related to altered psychophysiological responses to reinforcement. Biological Psychiatry, 68, 337-343.
Lykken, D. T. (1957). A study of anxiety in the sociopathic personality. Journal of Abnormal and Social Psychology, 55, 6-10.

Maren, S., \& Quirk, G. J. (2004). Neuronal signalling of fear memory. Nature Reviews Neuroscience, 5, 844-852.

Marsh, A. A., Finger, E. C., Mitchell, D. G. V., Reid, M., Sims, C., Kosson, D. S., et al. (2008). Reduced amygdala response to fearful expressions in children and adolescents with callousunemotional traits and disruptive behaviour disorders. American Journal of Psychiatry, 165, 712-720.

Matthys, W., \& Lochman, J. E. (2010). Oppositional defiant disorder and conduct disorder in childhood. Chichester: Wiley-Blackwell.

Matthys, W., van Goozen, S., de Vries, H., Cohen-Kettenis, P., \& van Engeland, H. (1998). The dominance of behavioural activation over behavioural inhibition in conduct disordered boys with and without attention deficit hyperactivity disorder. Journal of Child Psychology and Psychiatry, 39, 643-651.

Matthys, W., van Goozen, S. H. M., Snoek, H., \& van Engeland, H. (2004). Response perseveration and sensitivity to reward and punishment in boys with oppositional defiant disorder. European Child and Adolescent Psychiatry, 13, 362-364.

Matthys, W., Vanderschuren, L. J. M. J., \& Schutter, D. J. L. G. (2012). The neurobiology of Oppositional Defiant Disorder and Conduct Disorder: Altered functioning in three mental domains. Development and Psychopathology (in press).

Maughan, B., \& Rutter, M. (2001). Antisocial children grown up. In J. Hill \& B. Maughan (Eds.), Conduct disorders in childhood and adolescence (pp. 507-552). Cambridge: Cambridge University Press.

McCart, M. R., Priester, P. E., Davies, W. H., \& Azen, R. (2006). Differential effectiveness of behavioral parent-training and cognitive-behavioral therapy for antisocial youth: A metaanalysis. Journal of Abnormal Child Psychology, 34, 527-543.

Morgan, A. B., \& Lilienfeld, S. O. (2000). A meta-analytic review of the relation between antisocial behavior and neuropsychological measures of executive function. Clinical Psychological Review, $20,113-136$.

Nigg, J. T. (2006). What causes ADHD? Understanding what goes wrong and why. New York: Guilford.

Nock, M. K., Kazdin, A. E., Hiripi, E., \& Kessler, R. C. (2007). Lifetime prevalence, correlates, and persistence of oppositional defiant disorder: Results from the national Comorbidity Survey Replication. Journal of Child Psychology and Psychiatry, 48, 703-713.

O’Doherty, J. P., Buchanan, T. W., Seymour, B., \& Dolan, R. J. (2006). Predictive neural coding of reward preference involves dissociable responses in human ventral midbrain and ventral striatum. Neuron, 49, 157-166.

Oosterlaan, J., Logan, G. D., \& Sergeant, J. A. (1998). Response inhibition in $\mathrm{AD} / \mathrm{HD}, \mathrm{CD}$, comorbid $\mathrm{AD} / \mathrm{HD}+\mathrm{CD}$, anxious and normal children: A meta-analysis of studies with the stop task. Journal of Child Psychology and Psychiatry, 39, 411-426.

Patterson, G. R. (2002). The early development of coercive family process. In J. B. Reid, G. R. Patterson, \& J. Snyder (Eds.), Antisocial behavior in children and adolescents: A developmental analysis and model of intervention (pp. 25-64). Washington: American Psychological Association.

Pennington, B. F., \& Ozonoff, S. (1996). Executive functions and developmental psychopathology. Journal of Child Psychology and Psychiatry, 37, 51-87.

Phelps, E. A., \& LeDoux, J. E. (2005). Contributions of the amygdala to emotion processing: From animal models to human behaviour. Neuron, 48, 175-187.

Phillips, G. D., Setzu, E., \& Hitchcott, P. K. (2003). Facilitation of appetitive pavlovian conditioning by d-amphetamine in the shell, 
but not the core, of the nucleus accumbent. Behavioral Neuroscience, 117, 675-684.

Raaijmakers, M. A. J., Posthumus, J., van Hout, B., van Engeland, H., \& Matthys, W. (2011). Cross-sectional study into the costs and impact on family functioning of 4-year old children with aggressive behaviour. Prevention Science, 12, 192-200.

Raaijmakers, M. A. J., Smidts, D. P., Sergeant, J. A., Maassen, G. H., Posthumus, J. A., van Engeland, H., et al. (2008). Executive functions in preschool children with aggressive behaviour: Impairments in inhibitory control. Journal of Abnormal Child Psychology, 36, 1097-1107.

Reid, J. B., Patterson, G. R., \& Snyder, J. (2002). Antisocial behavior in children and adolescents. A developmental analysis and model of intervention. Washington: American Psychological Association.

Robbins, T. W., \& Everitt, B. J. (2007). A role for mesencephalic dopamine in activation: Commentary on Berridge (2006). Psychopharmacology (Berlin), 191, 433-437.

Robbins, T. W., Watson, B. A., Gaskin, M., \& Ennis, C. (1983). Contrasting interactions of pipradrol, d-amphetamine, cocaine, cocaine analogues, apomorphine and other drugs with conditioned reinforcement. Psychopharmacology (Berlin), 80, 113-119.

Rowe, R., Costello, E. J., Angold, A., Copeland, W., \& Maughan, B. (2010). Developmental pathways in oppositional defiant disorder and conduct disorder. Journal of Abnormal Psychology, 119, $726-738$.

Rubia, K. (2011). 'Cool' inferior frontostriatal dysfunction in attention-deficit/hyperactivity disorder versus 'hot' ventromedial orbitofrontal-limbic dysfunction in conduct disorder: A review. Biological Psychiatry, 69, e69-e87.

Rubia, K., Halari, R., Cubillo, A., Smith, A., Mohammed, A.-M., Scott, S., et al. (2010). Disorder-specific inferior prefrontal hypofunction in boys with pure attention-deficit/hyperactivity disorder compared to boys with pure conduct disorder during cognitive flexibility. Human Brain Mapping, 31, 1823-1833.

Rubia, K., Halari, R., Smith, A., Mohammed, M., Scott, S., \& Brammer, M. J. (2009a). Shared and disorder-specific prefrontal abnormalities in boys with pure attention-deficit/hyperactivity disorder compared to boys with pure $\mathrm{CD}$ during interference inhibition and attention allocation. Journal of Child Psychology and Psychiatry, 50, 669-678.

Rubia, K., Halari, R., Smith, A., Mohammed, M., Scott, S., Giampetro, V., et al. (2008). Dissociated functional brain abnormalities of inhibition in boys with pure conduct disorder and in boys with pure attention deficit hyperactivity disorder. American Journal of Psychiatry, 165, 889-897.

Rubia, K., Smith, A. B., Halari, R., Matsukara, F., Mohammad, M., Taylor, E., et al. (2009b). Disorder-specific dissociation of orbitofrontal dysfunction in boys with pure conduct disorder during reward and ventrolateral prefrontal dysfunction in boys with pure ADHD during sustained attention. American Journal of Psychiatry, 166, 83-94.

Schoemaker, K., Bunte, T., Wiebe, S. A., Espy, K. A., Dekovic, M., \& Matthys, W. (2012). Executive function deficits in preschool children with ADHD and DBD. Journal of Child Psychology and Psychiatry, 53, 111-119.

Schoenbaum, G., \& Roesch, M. (2005). Orbitofrontal cortex, associative learning, and expectancies. Neuron, 47, 633-636.

Schultz, W. (2007). Multiple dopamine functions at different time courses. Annual Review of Neuroscience, 30, 259-288.

Schutter, D. J. L. G., van Bokhoven, I., Vanderschuren, L. J. M. J., Lochman, J. E., \& Matthys, W. (2011). Risky decision making in substance dependent adolescents with a disruptive behaviour disorder. Journal of Abnormal Child Psychology, 39, 333-339.
Scott, S., Knapp, M., Henderson, J., \& Maughan, B. (2001). Financial costs of social exclusion: Follow-up study of antisocial children into adulthood. British Medical Journal, 323, 191-194.

Sijtsema, J. J., Veenstra, R., Lindenberg, S., van Roon, A. M., Verhulst, F. C., Ormel, J., et al. (2010). Mediation of sensation seeking and behavioral inhibition on the relationship between heart rate and antisocial behaviour: The TRAILS study. Journal of the American Academy of Child and Adolescent Psychiatry, 49, 493-502.

Snoek, H., van Goozen, S. H. M., Matthys, W., Buitelaar, J. K., \& van Engeland, H. (2004). Stress responsivity in children with externalising behavior disorders. Development and Psychopathology, 16, 389-406.

Stadler, C., Sterzer, P., Schmeck, K., Krebs, A., Kleinschmidt, A., \& Poustka, F. (2007). Reduced anterior cingulate activation in aggressive children and adolescents during affective stimulation: Association with temperament traits. Journal of Psychiatric Research, 41, 410-417.

Sterzer, P., Stadler, C., Krebs, A., Kleinschmidt, A., \& Poustka, F. (2005). Abnormal neural responses to emotional stimuli in adolescents with conduct disorder. Biological Psychiatry, 57, 7-15.

Sterzer, P., Stadler, C., Poustka, F., \& Kleinschmidt, A. (2007). A structural neural deficit in adolescents with conduct disorder and its association with lack of empathy. Neuroimage, 37, 335-342.

Stringaris, A., \& Goodman, R. (2009a). Three dimensions of oppositionality in youth. Journal of Child Psychology and Psychiatry, 50, 216-223.

Stringaris, A., \& Goodman, R. (2009b). Longitudinal outcome of youth oppositionality: Irritable, headstrong, and hurtful behaviours have distinctive predictions. Journal of the American Academy of Child and Adolescent Psychiatry, 48, 404-412.

Swanson, J., Kraemer, H. C., Hinshaw, S. P., Arnold, L. E., Conners, C. K., Abikoff, H. B., et al. (2001). Clinical relevance of the primary findings of the MTA: Success rates based on severity of ADHD and ODD symptoms at the end of the treatment. Journal of the American Academy of Child and Adolescent Psychiatry, 40, 168-179.

Taylor, E., Schachar, R., Thorley, G., Wieselberg, H. M., Everitt, B., \& Rutter, M. (1987). Which boys respond to stimulant medication? A controlled trial of methylphenidate in boys with disruptive behaviour. Psychological Medicine, 17, 121-143.

Tye, K. M., Tye, L. D., Cone, J. J., Hekkelman, E. F., Janak, P. H., \& Bonci, A. (2010). Methylphenidate facilitates learning-induced amygdala plasticity. Nature Neuroscience, 13, 475-481.

van Goozen, S. H. M., Cohen-Kettenis, P. T., Snoek, H., Matthys, W., Swaab-Barneveld, H., \& van Engeland, H. (2004). Executive functioning in children: A comparison of hospitalized ODD and ODD/ADHD children and normal controls. Journal of Child Psychology and Psychiatry, 45, 284-292.

van Goozen, S. H. M., Matthys, W., Cohen-Kettenis, P. T., Buitelaar, J. K., \& van Engeland, H. (2000). Hypothalamic-pituitaryadrenal axis and autonomic nervous system activity in disruptive children and matched controls. Journal of the American Academy of Child and Adolescent Psychiatry, 39, 1438-1445.

van Goozen, S. H. M., Matthys, W., Cohen-Kettenis, P. T., Gispen-de Wied, C., Wiegant, V. M., \& van Engeland, H. (1998). Salivary cortisol and cardiovascular activity during stress in oppositionaldefiant disorder boys and normal controls. Biological Psychiatry, 43, 531-539.

van Honk, J., Harmon-Jones, E., Morgan, B. E., \& Schutter, D. J. L. G. (2010). Social explosive minds: The triple imbalance hypothesis of reactive aggression. Journal of Personality, 78, 67-94.

Volkow, N. D., Wang, G. J., Fowler, J. S., Telang, F., Maynard, L., Logan, J., et al. (2004). Evidence that methylphenidate enhances 
saliency of a mathematical task by increasing dopamine in the human brain. American Journal of Psychiatry, 161, 1173-1180.

Webster-Stratton, C. (2001). The incredible years: Parents and children videotape series: A parenting course (BASIC). Seattle, WA: Incredible Years.

Wells, K. C., Lochman, J. E., \& Lenhart, L. A. (2008). Coping power parent group program: Workbook. New York, NY: Oxford.
Wyvell, C. L., \& Berridge, K. C. (2000). Intra-accumbens amphetamine increases the conditioned incentive salience of sucrose reward: Enhancement of reward "wanting" without enhanced "liking" or response reinforcement. Journal of Neuroscience, $20,8122-8130$. 